\title{
Measuring local and global vibration modes in model plants
}

\author{
Christelle Der Loughian ${ }^{\text {acc }}$, Loïc Tadrist ${ }^{\mathrm{a}}$, Jean-Marc Allain b, Julien Diener ${ }^{\mathrm{a}}$, \\ Bruno Moulia ${ }^{\mathrm{c}}$, Emmanuel de Langre ${ }^{\mathrm{a}}$, \\ ${ }^{a}$ Department of Mechanics, LadHyX, Ecole Polytechnique-CNRS, 91128 Palaiseau, France \\ ${ }^{\mathrm{b}}$ Department of Mechanics, LMS, Ecole Polytechnique-CNRS, 91128 Palaiseau, France \\ 'INRA, PIAF, 63100 Clermont-Ferrand, France
}

\begin{abstract}
Model plants are extensively used in biological studies, and their mechanical behaviour needs to be better understood, in relation to studies in mechanoperception for instance. We present here the first approach to derive experimentally the modal parameters of two of these plants, Arabidopsis thaliana and Populus tremula $\times$ alba. A classical sinusoidal sweep excitation is used, with a measurement of displacements based on LKT optical flow tracking, followed by a Bi-Orthogonal decomposition (BOD). This allows to estimate several modal frequencies for each plant, as well as the corresponding spatial localizations of deformation. Analyzing the modal frequencies, we show that global and local modes correspond to distinct ranges of frequencies and depend differently on plant size. Possible phenotyping applications are then discussed.
\end{abstract}

Key words: Vibration, Modes, Biomechanics, Image Correlation, Plants, Arabidopsis, Populus, BOD, LKT

\section{Introduction}

Plant biomechanics adresses issues related to a very large variety of organisms, from spores to trees, and takes both fundamental and applied points of view. In the development of plant biology, model plants play a key role as they allow to focus fundamental or applied studies on genomically sequenced organisms. Two of these model plants, Arabidopsis thaliana and Populus tremula $\times$ alba (referred to as At and Pt in the following) are often considered in fundamental biology studies where mechanical issues are involved [1]. Unfortunately, whereas these model plants are known in great detail in terms of genetics [2], little is known of their mechanical behaviour. To investigate issues such as the perception of deformation by plants (thigmomorphogenesis [3]) it becomes necessary to have a good view of how a plant deforms under

Email address: emmanuel.de-langre@polytechnique.edu (Emmanuel de Langre). 
external load. More precisely, vibrational characteristics such as modal frequencies are largely unknown while the dynamic response to excitations such as wind is known to affect growth.

In parallel, the dynamics of other plant species such as trees or crops have been extensively studied, see for instance in [4]. Generally only the first mode of vibration has been measured and modellized. This fundamental mode is known to carry a large part of the response to wind, but higher frequency modes have also been shown to play a important role in dissipation of motion [5]. This led recently to several experimental and theoretical studies on the multimodal dynamics of these multi-branched systems such as trees. One of the key recent results was that these higher modes are localized in space [6], a living illustration of the general concept of localization of branched systems [7]. For crops, such as wheat or alfalfa, little is known outside their fundamental mode of vibrations $[8,9]$.

At the growth stage where they are studied by biologists, model plants such as Pt or At do not have the same geometrical characteristics as fully grown trees which have up to 8 orders of regular branching. They however display very contrasted shoot architecture with different level of mechanical heterogeneities: young $\mathrm{Pt}$ trees used as model plants are made of a straight stem with large leaves, with the apical part (referred to as "apex" in the following) being made of much more compliant and soft tissues, figure 1a. In contrast, At at the inflorescence stage are generally composed of a few leaves close to the ground (with little possible deformations), but develop a branched floral stem (inflorescence) that is slender and subjected to deformation, see figure 1b. Note also that for biological studies, both At and Pt are usually grown in pots, with substrates (i.e. artificial soil) of weak cohesion.

Measuring the modal properties, such as frequencies and modal shapes, of such systems is a challenge. For large trees, standard modal methods of modal analysis have been used, such as excitation by pull and release or hammer impact, with accelerometers or strain gages, see in [4]. Here, in small model plants, tissues are soft, organs are very light, and the mechanical experiments should not be destructive or too invasive. Using a shaking table must be avoided because of the softness of the soil. Moreover, because of the large variability in geometry among plants of a given species at a given age, only statistical results have some meaning. This implies that a large number of experiments is undertaken, and therefore that the method used has to be simple enough.

The aim of the present paper is to present a new approach to measure modal quantities in such systems, and to give a first interpretation of the results in terms of potential localization of the modes and frequencies. The further application of this technique will also be discussed.

\section{Materials and Methods}

\subsection{Plants}

We consider two sets of plants, the young Poplar Populus tremula $\times$ alba and Arabidopsis thaliana at the stage where it develops a floral stem. More precisely, Hybrid polars ( $P$. alba $\times$ tremula clone INRA 717-1B4) were obtained by in vitro micropropagation and grown on nutrient solution [10]. These trees were grown in a greenhouse with controlled temperature and irrigated by subirrigation. The experiments are conducted on seven young unbranched poplars, figure 1a, taken at different stages of growth with total heights between $18 \mathrm{~cm}$ and $100 \mathrm{~cm}$. The Arabidopsis thaliana plants were grown in a greenhouse under a $16 \mathrm{~h} \mathrm{light} / 8 \mathrm{~h}$ dark regime at $21^{\circ} \mathrm{C}, 55 \%$ relative humidity, figure $1 \mathrm{~b}$. A set of $24 \mathrm{At}$ plants was used. The height of the main stem varied from $10 \mathrm{~cm}$ to $25 \mathrm{~cm}$. These two set of plants are kept under standard thermal conditions during the vibration experiments, by using low temperature LED lighting for the visual acquisition. 


\subsection{Excitation}

The proposed method is based on a sinusoidal excitation, with a slow sweep in frequency. Each plant is excited by a shaker attached close to the base of the stem, figure 1c. The shaker (PCB Piezotronics, $K 2007 E 01)$ is controlled by a low frequency generator (AIM-TTI, model TG2000). For most tests on Pt the applied load was measured by a force transducer (PCB Piezotronics, 288D01SN2715). Sweeps of 2 $\mathrm{Hz}$, lasting $30 \mathrm{~s}$, are used. The range of sweeps was chosen such that no more than one mode is found in a given sweep. As Pt and At have quite different masses and stiffness, the excitation has to be adapted in terms of range of frequencies: Pt were stimulated between 0 and $36 \mathrm{~Hz}$ (18 sweeps), and At between 0 and $24 \mathrm{~Hz}$ only (12 sweeps). The level of excitation, of the order of $0.1 \mathrm{~N}$, was also adapted to have small amplitude of motion, yet measurable. As only linear modal characteristics are sought, the magnitude of the force does not affect the results.

\subsection{Measurement of displacement}

The motion of the plants are recorded with a high speed camera (Vosskuhler HCC-1000BGE), using a high level of light with a LED system to avoid increasing the temperature of the plants. The frequency of capture is such that it is always more than 5 times the frequency of the excitation. A typical motion is illustrated in figure 2 by image superposition over a period. The movies corresponding to each sweep on a $2 \mathrm{~Hz}$ range are then processed to derive the displacement of material points during that sweep, using the Kineplant-CR toolbox in MATLAB (The MathWorks), detailed in [11], as follows. First, a set of about 200 materials points displaying high orthogonal gradients in grey-level intensity ("good features to track") is automatically selected using the Tomasi-Kanade method [12]. To improve the selection of points, based on local gradients of color intensity, additional marks were added on the At plants. These points are tracked from image to image using a multi-scale implementation of the Lucas-Kanade algorithm [13]. This allows to derive the displacement field evolution with time, denoted $X(M, t)$ where $M$ denotes the current material point.

To extract the dominant features of the movement, we then use for each sweep the same decomposition method as in $[9,11,14]$, based on Bi-Orthogonal Decomposition (BOD) [15], such that

$$
X(M, t)=\sum_{k} \alpha_{k} \Psi_{k}(M) \mu_{k}(t) .
$$

This unique decomposition yields a series of BOD modes, composed of a spatial part, $\Psi_{k}(M)$, called the topos and a temporal part, $\mu_{k}(t)$, called chronos. The amplitude coefficients $\alpha_{k}$ give the relative contribution of each BOD mode to the measured motion during the whole sweep. Note that the topos that are extracted by the BOD decomposition out of the evolution of displacement during a given sweep are not the normal modes of vibration of the system. Yet, as detailed below, if a resonance occurs in the sweep, the motion will be dominated by the response of the resonating normal mode of vibration, and the topos will be a measure of the modal shape.

\subsection{Identification of modes}

The identification of modes in a given frequency sweep is based on the assumption that if a modal frequency exists in the swept range the motion will be dominated by the resonant response on this particular mode. This assumes that dampings are not too large, and that the ranges of sweep can be made small enough that there exists at the most one mode in a given range. The results from the $\mathrm{Bi}$ Orthogonal Decomposition allow to quantify the dominance of one mode in the response: if one of the 
$\alpha_{k}$ clearly dominates the others, we assume that a single mode is present with a modal frequency in the intervals of the sweep, and a modal shape which is the corresponding topos $\Psi_{k}(M)$. The criterion used is that the $\alpha_{k}$ is 3 times (respectively 5 times) larger than the next one in magnitude, for At and Pt respectively. Conversely, if no dominant spatiotemporal structure appears in the response, it can be stated that there is no mode in that range of frequency. Figure 2 shows the dominant topos and chronos pair in a sweep from 0 to $2 \mathrm{~Hz}$ on a $\mathrm{Pt}$.

To give a finer estimate of the modal frequency, better than the two hertz range of the sweep, the chronos is then analysed. Because of the slow variation of frequency in the sweep, the variation with time of the amplitude of the chronos corresponds to the variation with frequency. A resonance peak is expected to exist at the modal frequency, with a shift of phase between the displacement function $\mu_{k}(t)$ and the applied load measured by the transducer. When these features are clearly detected, as in figure 2 , the modal frequency is set at that of the forcing at the time of the resonance. Otherwise the modal frequency is set at the mean value of the sweep range.

\section{Results}

\subsection{Modal densities}

The existence of a resonant mode in a given sweep can be represented as a probability. This mode probability can be estimated by the systematic analysis of 18 sweeps for each of the $7 \mathrm{Pt}$ trees, and 12 sweeps for each of the 24 At plants. A mode probability of 1 for a given sweep range means that all tested plants display a modal frequency in that range. Figure 3 shows that for $\mathrm{Pt}$, modes were found for all plants in almost all $2 \mathrm{~Hz}$ intervals, from 0 to $36 \mathrm{~Hz}$. For At plants, only a few intervals contained modes for a majority of plants.

These high modal densities are consistent with the results of previous theoretical and experimental work on trees and other branched systems, see for instance $[6,16,17]$ where such a density is usually associated with a localization of deformations in space.

\subsection{Global and local modes}

We now analyse the modal shapes $\Phi_{N}(M)$ corresponding to the modal frequencies. They are estimated as the spatial functions of the dominant mode $\Psi_{1}(M)$ of each interval, if there is one. A large variety of mode shapes is found, some involving deformation of the whole plant, and some of only part of the plant, as illustrated in figures 4 and 5. To quantify this spatial distribution of deformation in the plant, and access possible localization, we compute the norm of the displacement vector $\Phi_{N}(M)$ at each point $M$ as a function of the vertical coordinate of $M$ (see examples in figures 4 and 5). A simple criterion may then be used to classify these modes, as follows. For both At and Pt plants a mode is noted as global when more than $50 \%$ of points have a norm of the displacement larger than $33 \%$ of the maximum. This corresponds to modes involving essentially bending of the stem (in Pt) or of the main branches (in At), figures 4 and 5. Other modes are noted as local modes. For Pt, two types of these local modes are found. The first type involves only the tip of the tree, as illustrated in figure 4 (middle), and are referred to as apex modes. The second type have a localized deformation on individual leaves, figure 4 (bottom). For At plants, local modes involve the motion of small organs attached to the branches, called silica, figure 5 .

The mode probability for each type of mode is also given in figures 4 and 5. Evidently, for Pt, there is a direct link between the spatial localization and the range of frequency. The global modes are only found at very low frequency, about $1 \mathrm{~Hz}$. Frequencies of apex modes are in a separate, higher range, and those 
of leaf modes still higher. It is worth noting that this separation of frequencies is found by considering a set of $7 \mathrm{Pt}$ plants, covering a broad range of size and morphology. For the At plants the organisation of frequencies is somewhat different, as can be seen from their mode probability: global and local modes span across the whole range of frequencies, with a slight bias towards lower frequencies for global modes.

\subsection{Relation between modal frequency and plant size}

The results presented above did not take into account the large variety of size of plants in the two sets. To evidence the role of this parameter, we compute, for a given plant, the mean value of the frequencies of modes of each category as defined above. This mean frequency is shown as a function of the plant height in figure 6.

Here, the global and local modes differ statistically in their dependence on plant size. Figure 6 shows that the global modes of At and $\mathrm{Pt}$ decrease with plant size (the $R^{2}$ determination coefficient over a power law being of 0.91 for $\mathrm{Pt}$ and of 0.36 for At). However the dependence with size is quantitatively very distinct: weaker in $\mathrm{Pt}$ that in At. These two variations fall between two theoretical dependance that are shown on the same graph, based on the assumption that the frequency is essentially affected by geometrical effects [6]. For a beam of diameter $D$ and length $L$ the frequencies of the bending modes of vibration vary as $D / L^{2}$. This can be generalysed to non uniform systems in bending, see [6], where $D$ and $L$ are the diameter and height scales respectively. Therefore, a dependence in $L^{-2}$ corresponds to plants that are homothetic in height only with identical diameters. Alternatively, a dependence of frequencies as $L^{-1 / 2}$ is associated to plants where length and diameter are linked by the often described allometric law where $D$ varies as $L^{3 / 2}[6]$. The two model plants we have considered here have a dependence of frequencies on size that fall between these two models, At being closer to the first and Pt to the second one. This is confirmed by plotting, figure 6 , the base diameter of all plants as a function of their height.

For local modes, the correlation with plant size are weaker, with $R^{2}$ coefficients 4.2 to 2.8 times smaller than for the global modes in Pt (for apex and leaves respectively), and 6.6 times smaller in At (for silica). In fact, local systems such as the apex, or the set of leaves or fruits (silica), are rather similar from plants to plants, and their mechanical characteristics do not depend on the size of the plants. Local modes are therefore not expected to depend on the height of the plant.

\section{Discussion and conclusion}

We have shown that modes of small model plants may be measured, both in terms of frequency and mode shapes. It should be noted that we have only analyzed here the modal shapes in their projection on a plane orthogonal to the axis of view. However, tests with a rotation of the plant gave the same sets of modes, which means that all modes have a three dimensional motion. To obtain the full modal shape in three dimensions, the same procedure can be done using a stereo acquisition and 3D reconstruction. Other modal quantities such as modal damping or modal stiffness are also measurable but would require finer data on the transfer function.

Although the plants of a given species differed largely from one to another, due to natural variability, we obtained some relevant statistical information on how modes are localized in frequency and space. The most striking feature is that modes are indeed localized in these plants, although they are not well-defined branched systems. This localization of modes in organs such as leaves, apex or silica, is probably due to the large range of bending stiffness that exists in a given plant. For instance an order of magnitude of variation exists between the stem and a petiole. 
These results may now be used in two directions. First, we know now which part of the plant is deformed in a given frequency range. The localization of deformation gives a basis to look for mechanosensitive tissues in the plant. This raises the question of the perception of frequencies of deformation by plants. A second possible application is in the vast domain of phenotyping [18]: the modal characteristics of a system do depend on mass, stiffness, and geometry, and may therefore be used to assess the potential variation of these parameters with genetics. The method proposed in the present paper has many advantages, being non destructive and easily rendered automatic.

\section{Acknowledgements}

The authors thank Pascal Hémon, from Ecole polytechnique, for considerable help in the experiments, and Jean-Marie Frachisse and Tiffanie Giraut, from ISV, CNRS, Gif-sur-Yvette, for providing the At plants. This work has been supported by the grant AN5-09-BLAN-0245-01 from the Agence Nationale de la Recherche (project Senzo).

\section{References}

[1] L. Martin, N. Leblanc-Fournier, J.-L. Julien, B. Moulia, C. Coutand, Acclimation kinetics of physiological and molecular responses of plants to multiple mechanical loadings, Journal of Experimental Botany 61 (2010) 2403-2412.

[2] Arabidopsis Genome Initiative, Analysis of the genome sequence of the flowering plant arabidopsis thaliana., Nature 408 (6814) (2000) 796.

[3] M. J. Jaffe, Thigmomorphogenesis: the response of plant growth and development to mechanical stimulation, Planta 114 (1973) 143-157.

[4] E. de Langre, Effects of wind on plants, Annual Review of Fluid Mechanics 23 (2008) 971-979.

[5] B. Theckes, E. de Langre, X. Boutillon, Damping by branching: a bioinspiration from trees, Bioinspiration \& biomimetics 6 (4) (2011) 046010.

[6] M. Rodriguez, E. de Langre, B. Moulia, A scaling law for the effects of architecture and allometry on tree vibration modes suggests a biological tuning to modal compartmentalization, American Journal of Botany 95 (2008) $1523-1537$.

[7] M. Filoche, S. Mayboroda, Universal mechanism for Anderson and weak localization, Proceedings of the National Academy of Sciences 109 (37) (2012) 14761-14766.

[8] T. Farquhar, J. Z. Wood, J. van Beem, The kinematics of wheat struck by a wind gust, Journal of applied mechanics Transactions of the ASME 67 (2000) 496-502.

[9] C. Py, E. de Langre, B. Moulia, A frequency lock-in mechanism in the interaction between wind and crop canopies, Journal of Fluid Mechanics 568 (2006) 425-449.

[10] J. Morizet, M. Mingeau, Effect of environment on water-uptake, as studied on beheaded exudating tomato. 1. Role of nutrients, Annales agronomiques 27 (1976) 183-205.

[11] A. Barbacci, J. Diener, P. Hémon, B. Adam, N. Donès, L. Reveret, B. Moulia, A robust videogrametric method for the velocimetry of wind-induced motion in trees, Agricultural and Forest Meteorology, in press (2013).

[12] J. Shi, C. Tomasi, Good features to track, in: Computer Vision and Pattern Recognition, 1994. Proceedings CVPR'94., 1994 IEEE Computer Society Conference on, IEEE, 1994, pp. 593-600.

[13] J.-Y. Bouguet, Pyramidal implementation of the Lucas Kanade feature tracker: Description of the algorithm, Intel Corporation - Microprocessor Research Labs (2001) 5.

[14] C. Py, E. de Langre, B. Moulia, P. Hémon, Measurement of wind-induced motion of crop canopies from digital video images, Agricultural and Forest Meteorology 130 (2005) 223-236. 
[15] N. Aubry, R. Guyonnet, R. Lima, Spatiotemporal analysis of complex signals: theory and applications, Journal of Statistical Physics 64 (3-4) (1991) 683-739.

[16] M. Rodriguez, S. Ploquin, B. Moulia, E. de Langre, The multimodal dynamics of a walnut tree: Experiments and models, Journal of Applied Mechanics 79 (4) (2012) 4505.

[17] A. Leung, Dynamic substructure method for elastic fractal structures, Computers \& Structures 89 (3) (2011) 302-315.

[18] R. T. Furbank, M. Tester, Phenomics-technologies to relieve the phenotyping bottleneck, Trends in plant science 16 (12) (2011) 635-644. 


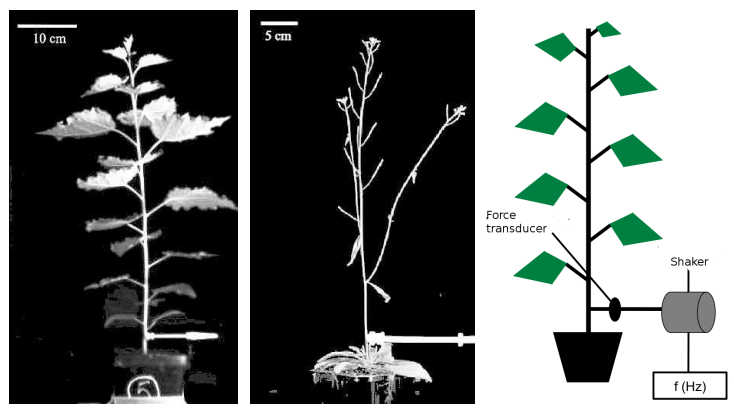

Figure 1. Model plants and experimental set-up. Left: Young poplar Populus tremula $\times$ alba, denoted Pt in the text. Center: Floral stem of Arabidopsis thaliana denoted At in the text. Right: schematic view of the excitation by the shaker. 

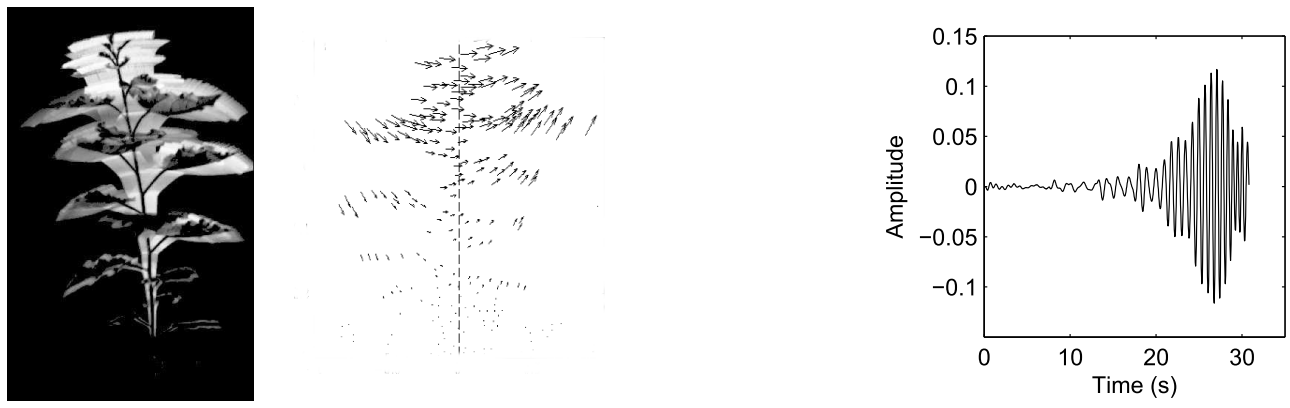

Figure 2. Left: Visualization of the oscillating motion using image superposition. Center and right: Example of the result of a BOD decomposition. Dominant spatial structure, or chronos (center), and the corresponding temporal structure, or topos (right). The peak of the chronos corresponds to a resonance and allows the identification of the modal frequency. 

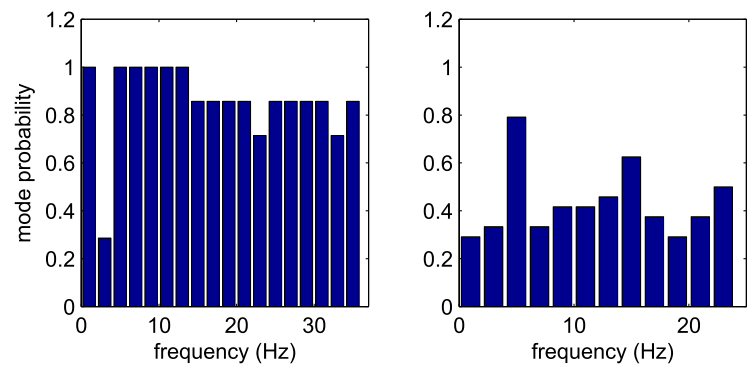

Figure 3. Mode probability in the two plant populations for each range of sweep. Left: Pt. Right: At. 

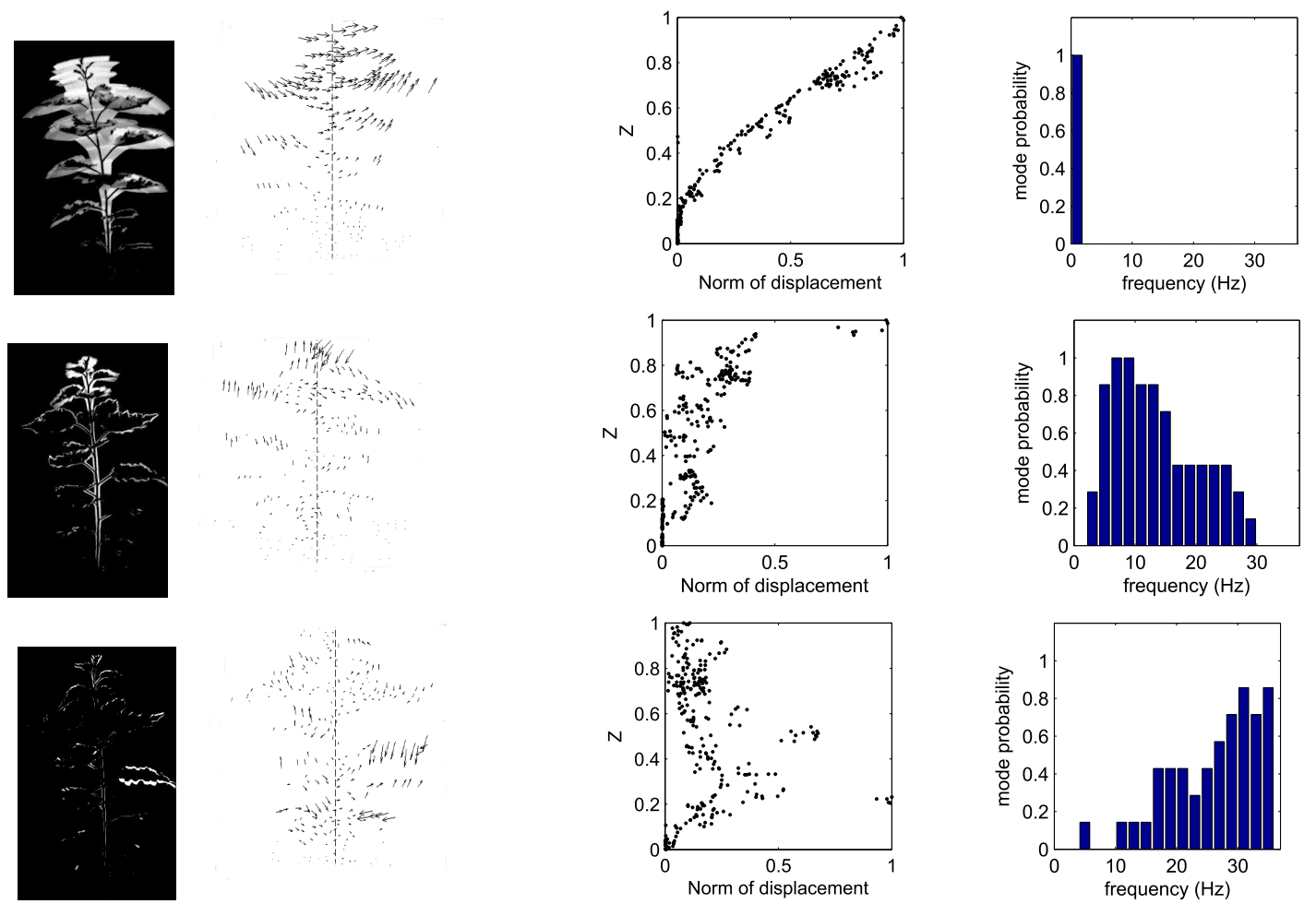

Figure 4. Localization of modes and frequencies in Pt. From top to bottom: global stem modes, local apex modes, local leaf modes. From left to right: for a given mode type, visualization of a typical mode shape by superposition, modal displacement field and norm of the modal displacement along the normalized plant height, and mode probability of that type. The frequency of the modes shown to exemplify each type are respectively $1.7,19$ and $31 \mathrm{~Hz}$ 

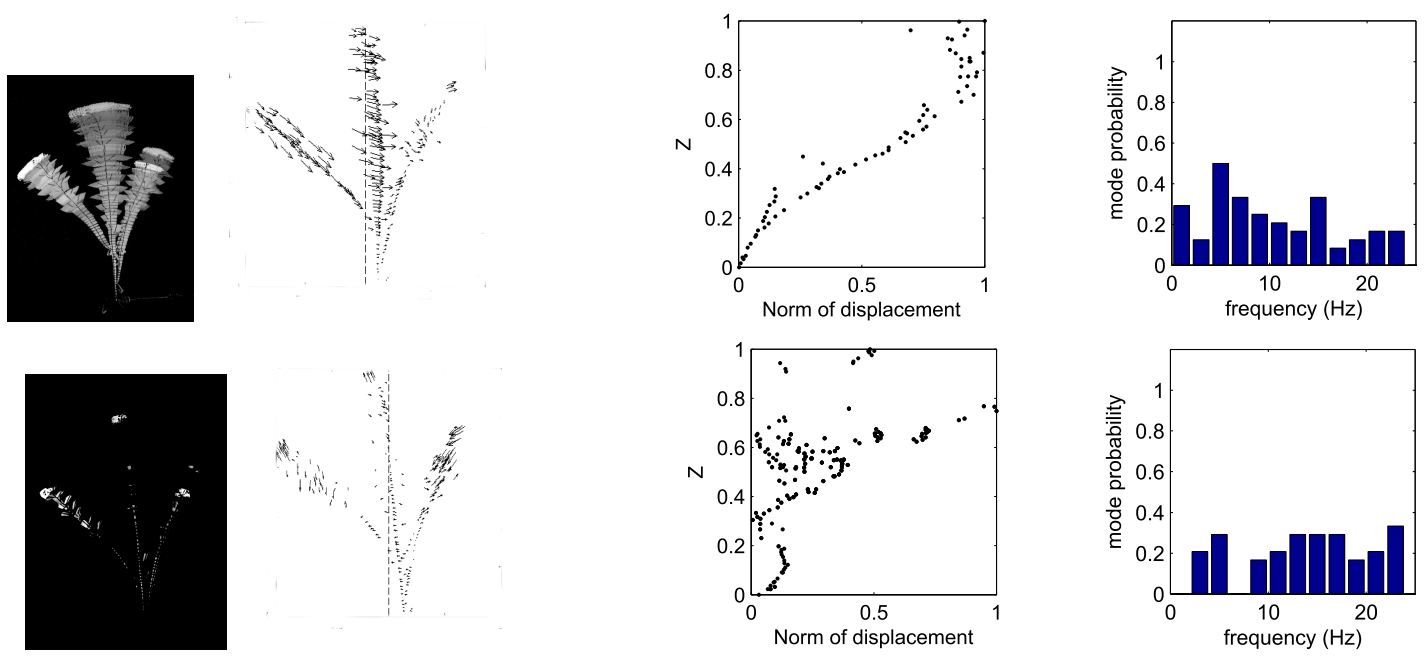

Figure 5. Localization of modes and frequencies in At, with the same presentation as in figure 4 . The modal frequencies are here 3 and $15 \mathrm{~Hz}$ 

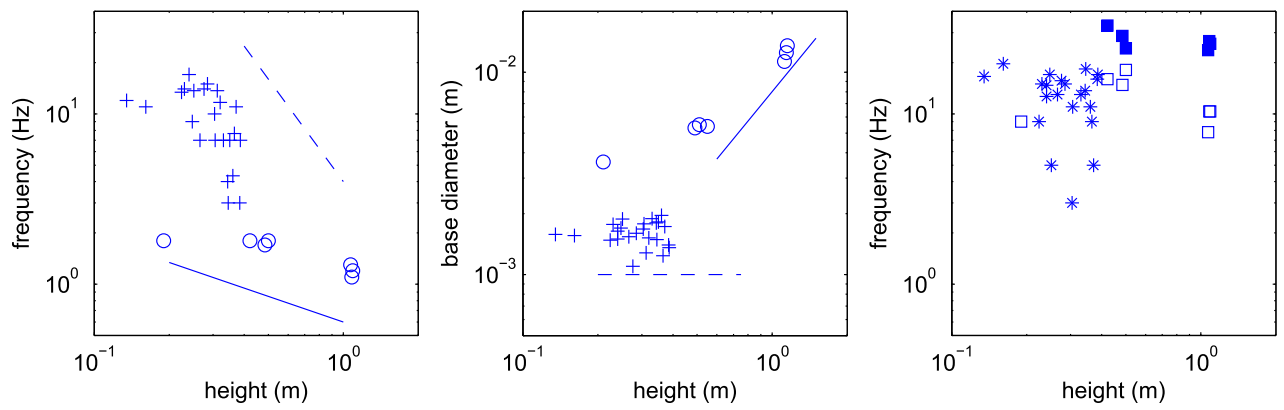

Figure 6. Effect of plant size on frequencies. Left: global modes of At $(+)$ and Pt (o). The dashed and solid lines correspond respectively to the -2 and $-1 / 2$ slopes of common models, see text. Center: Height and base diameters of At $(+)$ and $\mathrm{Pt}$ (o). The dashed and solid lines correspond respectively to the 0 and $3 / 2$ slopes of the same models. Right: local modes in At $(*)$ and in Pt $(\square$, apex modes and $\mathbf{\square}$, leaf modes). 